\section{ANÁLISIS DE VULNERABILIDAD ANTE AMENAZAS DE ORIGEN HÍDRICO EN LA CUENCA DEL ARROYO LAS TURBIAS, ARGENTINA}

Mario Gardiol ${ }^{1 *}$, María del Valle Morresi ${ }^{1}$, Lucila Grand ${ }^{1}$, Mercedes Cardoso ${ }^{2}$ y Norma Finelli ${ }^{2}$

\section{RESUMEN}

Las amenazas de origen hídrico suelen ocasionar consecuencias más o menos graves en un territorio según el nivel de vulnerabilidad que posea. La cuenca hidrográfica se considera como unidad de gestión, donde los organismos gubernamentales deben considerar los potenciales riesgos para planificar el ordenamiento territorial. En este sentido, para contribuir a la toma de decisiones apropiadas, es relevante conocer la vulnerabilidad y las variables que la integran. Para el caso de la cuenca del arroyo Las Turbias, provincia de Santa Fe (Argentina), se seleccionaron 19 variables que componen las dimensiones política-institucional, socio-económica y ambiental. Se utilizó la metodología multicriterio para establecer el orden de vulnerabilidad en los sectores de la cuenca. El proceso metodológico requirió de información primaria y secundaria. Se consideraron relevantes las entrevistas realizadas a los referentes de instituciones, vecinos y productores agropecuarios. Los resultados indican diferencias en la condición de vulnerabilidad entre los sectores de la cuenca para las dimensiones socio-económica y ambiental. En cuanto a la vulnerabilidad total, se observó que la cuenca inferior presentó mayor vulnerabilidad que los otros sectores de la cuenca. A partir de este análisis se podrían identificar las variables sobre las cuales tomar decisiones para mejorar la condición de vulnerabilidad.

\section{PALABRAS CLAVES}

Vulnerabilidad, Cuenca, Multicriterio, Desastres, Argentina

\section{VULNERABILITY ANALYSIS TO WATER-RELATED HAZARDS IN LAS TURBIAS BASIN, ARGENTINA}

\section{ABSTRACT}

Water-related hazards may trigger disasters depending on different people's and territories' level of vulnerability. Hydrographic basins are considered as a management unit, where government should consider all potential hazards and risks within territorial planning. In this sense, and to contribute to informed decision-making, it is fundamental to study vulnerability and all its variables. In Las Turbias basin, Santa Fe province (Argentina), 19 variables were selected: constituting the political-institutional, socio-economic, and environmental dimensions. The multicriteria methodology was used to establish an order of vulnerability in all sectors of the basin. The methodological process required primary and secondary information. Interviews to key actors from institutions, neighborhood organizations, and agricultural producers were carried out. The results indicate differences in vulnerability conditions among different basin sectors for the socio-economic and environmental dimensions. Regarding to overall vulnerability, the study points out that the lower-basin sector is more vulnerable than other sectors. Finally, authors discuss how the studied variables and measures could be considered by different stakeholders to reduce the vulnerability in Las Turbias basin.

\section{KEYWORDS}

Vulnerability, Basin, Multi-criteria, Disasters, Argentina
1. Facultad de Ingeniería y Ciencias Hídricas (FICH), Universidad Nacional del Litoral (UNL), Santa Fe, Argentina.

2. Facultad de Humanidades y Ciencias (FHUC), Universidad Nacional de Litoral (UNL), Santa Fe, Argentina.

*Autor de correspondencia: mariogardiol@gmail.com

Identificador:

http://revistareder.com/ handle-0719-8477-2020-078

\section{RECIBIDO}

15 de septiembre de 2020

\section{ACEPTADO}

2 de noviembre de 2020

\section{PUBLICADO}

1 de enero de 2021

\section{Formato cita}

Recomendada (APA): Gardiol, M., Morresi, M.V. Grand, L., Cardoso, M. \& Finelli, N. (2021). Análisis de Vulnerabilidad ante Amenazas de Origen Hídrico en la Cuenca del Arroyo Las Turbias, Argentina. Revista de Estudios

Latinoamericanos sobre Reducción del Riesgo de Desastres REDER, 5(1), 7086. http://revistareder.com/ handle-0719-8477-2020-078

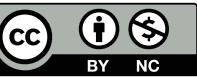

Todos los artículos publicados en REDER siguen una política de Acceso Abierto y se respaldan en una Licencia CreativeCommons Atribución-NoComercial 4.0 Internacional.

Revista de Estudios Latinoamericanos sobre Reducción del Riesgo de Desastres (REDER)

Diseño: Lupe Bezzina 


\section{INTRODUCCIÓN}

En los estudios orientados a planificar la reducción de riesgos de los territorios, el análisis de la vulnerabilidad emerge como tema central, complementado con el conocimiento de la amenaza y resiliencia. La valoración de la vulnerabilidad de un territorio, incluyendo las dimensiones y variables que la conforman es imprescindible para la evaluación de los riesgos que se desean gestionar.

En este sentido en la Argentina, se publicó el Documento País (2012) que proporciona una guía de referencia para todos los actores implicados en la gestión de riesgo de desastres en el país. En este documento se especifica el marco legal y normativo nacional, los actores institucionales, las condiciones de vulnerabilidad, las principales amenazas y escenarios de riesgo, entre otros puntos.

En octubre de 2016, se crea el Sistema Nacional para la Gestión Integral del Riesgo (SINAGIR) tras la sanción de la Ley $N^{\circ} 27287$. El mismo, está integrado por el Consejo Nacional para la Gestión Integral del Riesgo y la Protección Civil, por el Consejo Federal de Gestión Integral del Riesgo y la Protección Civil y por la Secretaría Ejecutiva. Tiene como objetivo articular el funcionamiento de los organismos del gobierno nacional, los gobiernos provinciales, de la Ciudad Autónoma de Buenos Aires y municipales, las organizaciones no gubernamentales y la sociedad civil, para fortalecer y optimizar las acciones destinadas a la reducción de riesgos, el manejo de la crisis y la recuperación.

Posteriormente, en el año 2017, la Secretaría de Protección Civil y Abordaje Integral de Emergencias y Catástrofes perteneciente al Ministerio de Seguridad publicó el manual para la elaboración de mapas de riesgo. Según Renda et al. (2017), el manual proporciona las bases para definir escenarios de riesgo representativos de cada realidad territorial, que contribuyan a la Gestión Integral del Riesgo de Desastres (prevención y mitigación) con un sentido federal amplio.

Por su parte la provincia de Santa Fe realizó diferentes acciones, una de ellas fue la publicación del atlas de riesgo por inundaciones de la provincia de Santa Fe (Gobierno de Santa Fe, 2012); cuyo objetivo es reducir las vulnerabilidades de la provincia de Santa Fe ante fenómenos hidrometeorológicos. En el mismo se detalla que las principales amenazas relacionadas a inundaciones tienen diferentes orígenes: por precipitaciones extremas, por desborde y por elevación de las napas freáticas.

En las últimas décadas en la Universidad Nacional del Litoral (UNL), se han realizado estudios relacionados a la temática del riesgo hídrico pero sin considerar a la cuenca hidrográfica como unidad de área de estudio.

El presente artículo es producto del desarrollo del proyecto de investigación CAID 2016 denominado "Riesgo asociado a fenómenos de origen hídrico en la cuenca del arroyo Las Turbias, provincia de Santa Fe, en el contexto de la variabilidad y el cambio climático regional" desarrollado en la Facultad de Ingeniería y Ciencias Hídricas (FICH) de la Universidad Nacional del Litoral (UNL).

El área de estudio es la cuenca del arroyo Las Turbias, una de las cuencas de aporte al arroyo Carrizales-Monje. Se encuentra localizada en el sector centro-sur de la provincia de Santa Fe. El relieve santafesino es una extensa llanura inclinada en dirección noroeste-sudeste, posee zonas muy planas especialmente en la parte norte de la provincia. Al sur de la provincia se encuentra la pampa ondulada con cotas que varían entre $10 \mathrm{~m}$ y $120 \mathrm{~m}$ s.n.m.

La cuenca ocupa una superficie aproximada de $971 \mathrm{~km}^{2}$ (ver Figura 1) y atraviesa los departamentos Belgrano (distritos, Las Rosas, Montes de Oca y Bouquet), Iriondo (distrito Clason), San Jerónimo (distritos Centeno, San Genaro y Casalegno) y San Martín (distritos María Susana, El Trébol y Los Cardos). Las localidades más próximas a la misma son hacia el norte María Susana, Los Cardos y Centeno, al oeste con Bouquet, al sur con Montes de Oca, Las Rosas y San Genaro y al este con Díaz y Casalegno. Las principales vías de comunicación de la cuenca son las rutas nacionales № 34 y las rutas provinciales № 13, 20 y 65 (Gardiol et al, 2018).

El arroyo Las Turbias posee una longitud de $76,5 \mathrm{~km}$. Este sistema hídrico se puede dividir en 3 sectores, siendo la subdivisión expuesta la adoptada por el Comité de cuenca (organismo que administra las obras hídricas a realizar). La cuenca superior se extiende desde la naciente hasta la ruta provincial $N^{0} 13$, posee una superficie de $611 \mathrm{~km}^{2}$, cota máximas y mínimas de 117 
y $85 \mathrm{~m}$ (Instituto Geográfico Nacional o IGN) respectivamente, pendiente promedio de $1,1 \mathrm{~m} / \mathrm{km}$ y una importante red de canales y bajos naturales (paleocañadas) que generan anegamientos prolongados en períodos de lluvias importantes.

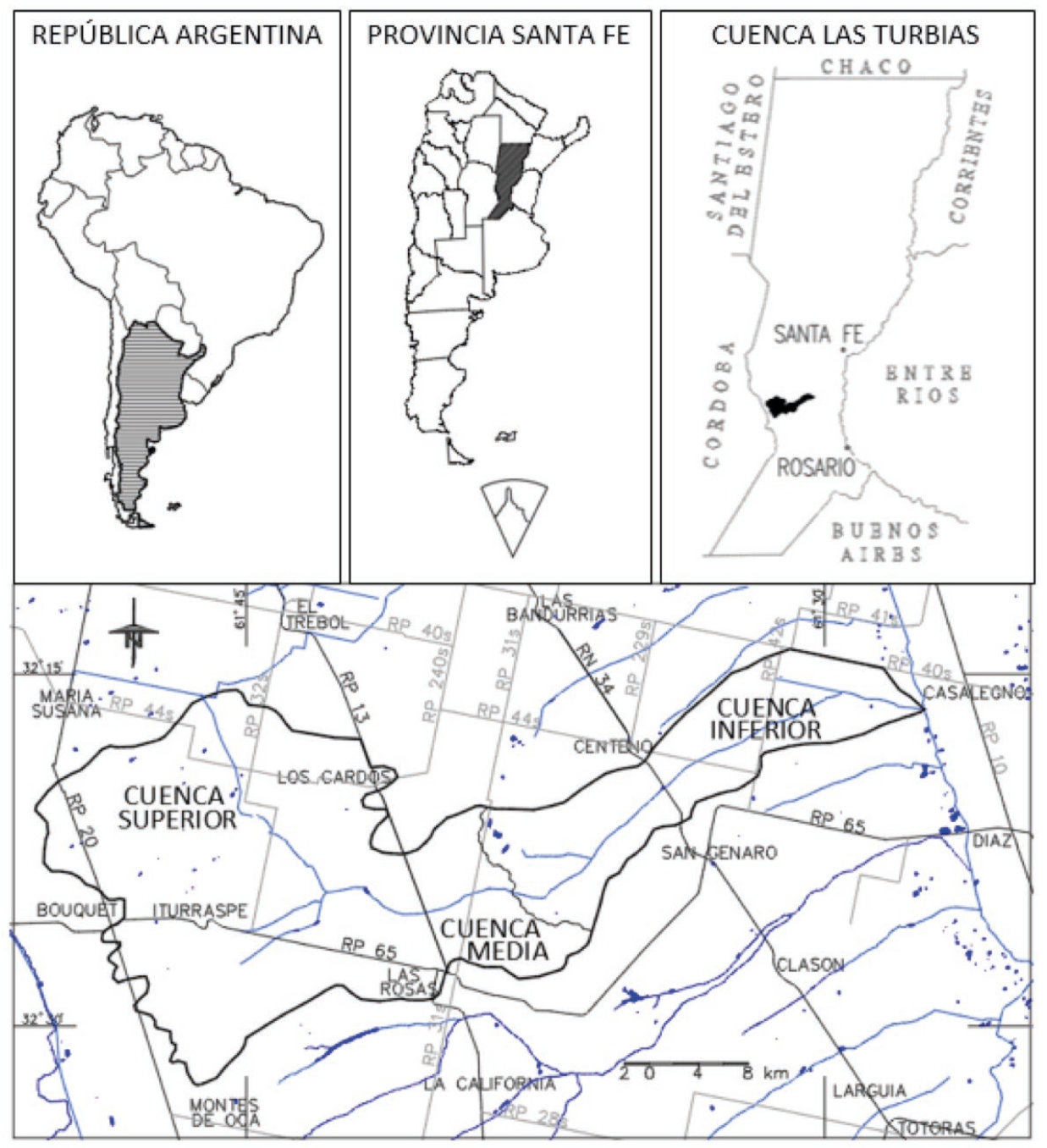

Figura 1. Cuenca del arroyo Las Turbias, Santa Fe, Argentina

Fuente: Autores, 2021

La cuenca media se extiende desde la ruta provincial $\mathrm{N}^{0} 13$ hasta la altura topográfica de $72,50 \mathrm{~m}$ (IGN) cuya superficie es de 100 km², cota máximas y mínimas de 105 y 72,50 m (IGN) respectivamente, pendiente promedio de $5 \mathrm{~m} / \mathrm{km}$ en un tramo de $8 \mathrm{~km}$ con escurrimiento de mayor velocidad. La cuenca inferior se extiende desde la cota de $72,50 \mathrm{~m}$ hasta la desembocadura del arroyo, donde su superficie es de $260 \mathrm{~km}^{2}$, cota máximas y mínimas de 72,50 y $30 \mathrm{~m}$ (IGN) respectivamente, pendiente promedio de $1,5 \mathrm{~m} / \mathrm{km}$, con escurrimiento definido en el cauce principal y paleocañadas paralelas al mismo.

Las precipitaciones anuales varían entre $975 \mathrm{~mm}$ y $1075 \mathrm{~mm}$. No obstante, en los últimos 15 años la cuenca presentó desbordes y anegamientos prolongados causados por eventos lluviosos intensos con recurrencia de 2 a 5 años (amenaza natural) y por intervenciones individuales, no planificadas (no controladas) y desordenadas, poniendo en discusión los límites de la cuenca en condiciones extremas. En menor grado se producen erosiones en márgenes de cauces y obras civiles. La amplia red vial interna de la cuenca, oficia como límite topográfico al escurrimiento de las aguas, -y en otras partes de conductores-, a lo que se suma el estado de obsolescencia y/o subdimensionamiento de las obras de drenaje.

Predominan suelos de alta capacidad productiva (clases I y II de capacidad de uso) predominantemente agrícola $(50 \%)$ y mixto agrícola-ganadero $(20 \%)$, siendo los principales cultivos soja, maíz y trigo (Castignani, 2011). 
En el aspecto demográfico las localidades más próximas poseen la siguiente población: Bouquet (1157), Casalegno (270), Centeno (2934), Las Rosas (13689), Los Cardos (1217), María Susana (3147), Montes de Oca (2609) y San Genaro (8266).

El objetivo de este trabajo es reconocer el orden de vulnerabilidad ante fenómenos de origen hídrico en la cuenca del arroyo Las Turbias. Para ello, se empleó una metodología de diseño mixto: cualitativa (basada en el uso de entrevistas a informantes claves) y cuantitativa (a partir del empleo de datos de fuentes directas e indirectas: censos, imágenes de satélite, etc.). Se recurrió a herramientas de metodologías multicriterio para analizar las tres dimensiones de vulnerabilidad: política-institucional, socio-económica y ambiental, las cuales ofrecen alternativas para los tomadores de decisión. Se identificaron los sectores de la cuenca mejor posicionados para gestionar el riesgo ante fenómenos de origen hídrico.

\section{MARCO TEÓRICO}

En el estudio del riesgo y los desastres se pueden reconocer distintas perspectivas que pudieron dar como resultado trabajos de corte físico, social y sistémicos. El desarrollo de las investigaciones fue evolucionando hacia la incorporación de factores antrópicos a los naturales tradicionalmente considerados, poniendo el acento cada vez más en el componente social.

Los primeros trabajos para el manejo de inundaciones que incorporan el factor humano son los de Gilberto White y sus colegas (Robert Kates e lan Burton). Realizado en la Universidad de Chicago, Estados Unidos entre 1940 y 1980, se trata de un estudio social de los desastres, derivado de las teorías funcionalistas de la Ecología Humana, promovidas por Harlen Barrows. Una de las ideas centrales de la teoría de White, es que las amenazas y los riesgos son producto de inadecuadas relaciones de convivencia entre la sociedad y su ambiente. El aspecto de la relación sociedad-ambiente y las formas de ajuste y aprovechamiento del medio para el desarrollo será un punto de esta teoría que retomarán autores de las otras corrientes (como Wilches-Chaux, Blaikie, Westgate, Wisner, Caputo y Herzer, entre otros).

La denominada "escuela de la vulnerabilidad" surge en los '70 del siglo pasado, donde el argumento principal es que los desastres son el resultado de procesos económicos y sociales globales, regionales y locales, que crean condiciones de existencia humana insostenibles frente a los eventos naturales extremos. Blaikie et al. (1996) ampliaron estos estudios de la vulnerabilidad al ámbito latinoamericano y asiático, aportando una significativa teoría social, con énfasis en la respuesta y recuperación de la comunidad luego del desastre.

Autores como Andrew Maskrey y Gilberto Romero, entre 1982 y 1983 realizaron un trabajo sobre la vulnerabilidad y el riesgo que estaba generando el proceso de desarrollo urbano, con la hipótesis de que esta dinámica respondía a procesos particulares de urbanización dependiente en América Latina. Se asume que la vulnerabilidad, como un factor condicionante de los desastres es socialmente construida; debe hacerse un manejo popular de los desastres, conociendo la vulnerabilidad y planificando la mitigación. Al mismo tiempo, compartiendo la premisa central de que los desastres no son naturales, sino que el factor humano incide constantemente, se sostiene que la vulnerabilidad es una construcción social y que "las amenazas son producto de una modalidad particular de desarrollo y sus formas de producción, consumo, distribución, asentamiento, expropiación de la naturaleza, etc" (Lavell, 2004: 8).

Diversos autores desarrollaron sus aportes a partir de un eje común: la consideración integral y holística del riesgo: en Colombia, Omar Cardona, Velasquez y Meyer, Wilches-Chaux; en México, Allan Lavell; en Bolivia, Argentina y otros países del cono sur, Caputo, Hardoy, Herzer (dentro de la órbita de CLACSO); estos trabajos que durante la década de los ' 80 y '90, los reunía en una perspectiva teórica compartida, derivaron en la conformación de LA RED: Red de Estudios Sociales en Prevención de Desastres en América Latina.

A partir de estos aportes teóricos, es posible definir los conceptos centrales de la categoría teórica del riesgo. Se denomina riesgo a "cualquier fenómeno de origen natural o humano que signifique un cambio en el medio ambiente que ocupa una comunidad determinada, que sea vulnerable a ese fenómeno" (Wilches-Chaux, 1993: 17). El riesgo, en el ámbito de la probabilidad, se constituye por la suma de dos elementos: amenaza (o peligro) y vulnerabilidad (que atañe a la población). Así, la existencia del riesgo dependerá de que el lugar en donde se manifieste esté ocupado o no por una comunidad vulnerable al mismo. La existencia de la amenaza dependerá del 
grado de probabilidad de su ocurrencia en esa comunidad. El desastre es la consecuencia de la manifestación de una amenaza en un territorio particularmente vulnerable a la misma.

La concepción de la vulnerabilidad también ha variado con el transcurso del tiempo. Del análisis de la vulnerabilidad de las construcciones ambientales enfatizando en sus componentes biofísicos, en las últimas décadas se han ido incorporando las vulnerabilidades creadas socialmente, aquellas que anteriormente habían sido completamente ignoradas. "Por vulnerabilidad vamos a denotar la incapacidad de una comunidad para 'absorber', mediante el autoajuste, los efectos de un determinado cambio en su medio ambiente, o sea su 'inflexibilidad' para adaptarse a ese cambio. (Wilches-Chaux, 1993).

La perspectiva de Cutter (2006) examina la vulnerabilidad, su estructura causal, su variabilidad espacial y el método para su reducción. Es la interacción entre naturaleza y sociedad lo que produce la vulnerabilidad de los lugares, dispositivo que cambia constantemente. Esta versión dinámica, cíclica de la vulnerabilidad, poco tiene que ver con definiciones estáticas de condiciones pre-existentes o predispositivas a las amenazas del ambiente.

En cuanto a la relación vulnerabilidad - resiliencia, existen autores que asumen que la primera incluye a la segunda (Vargas, 2002; Foschiatti, 2009); viceversa, o que ambos conceptos son independientes, pero se relacionan (Cutter et al., 2008). Luego, otros autores definen vulnerabilidad con los mismos términos que resiliencia (Blaikie et al., 1996).

La vulnerabilidad, concepto central de este trabajo, se constituye de diversas aristas. Para el abordaje de su complejidad Wilches Chaux (1993) se refiere a la vulnerabilidad global, entendida como una interacción de factores y características (internas y externas) que convergen en una comunidad particular y que conforman ese sistema dinámico. Ese conjunto de factores genera el bloqueo o incapacidad de la comunidad para responder adecuadamente ante la presencia de un riesgo determinado. Son diversas las dimensiones que componen la vulnerabilidad global, de acuerdo a los aportes de Wilches Chaux (1993): natural, física, económica, social, política, técnica, ideológica, cultural, educativa, ecológica e institucional.

La vulnerabilidad 'natural', está relacionada con los ecosistemas y sus actividades fundamentales (servicios ambientales o ecosistémicos).

La vulnerabilidad 'física', se expresa por la ubicación en las áreas propensas y las deficiencias de resistencias de los elementos expuestos a un riesgo. Un ejemplo de ello es la ubicación de viviendas en áreas bajas (inundables) o en zonas de deslizamientos de tierra en las laderas de montañas.

La vulnerabilidad 'económica', está relacionada a la capacidad adquisitiva de la población, por tanto la escasez de recursos actúa en contra, ya sea para la preparación ante un desastre como para la recuperación necesaria luego de la ocurrencia del evento. Las condiciones de pobreza aumentan la vulnerabilidad desde la mirada económica: esto se puede visualizar en el desempleo, los bajos ingresos, el acceso a los servicios, etc. (vulnerabilidad estrechamente vinculada a la social).

La vulnerabilidad 'social', se vincula con la capacidad de absorber y reponerse de las consecuencias de un desastre, por las formas de integración y los lazos de relacionamiento entre sus habitantes. Los grupos sociales pueden ser más o menos vulnerables con relación a cómo son capaces de organizarse y colaborar entre sus miembros.

La vulnerabilidad 'política', a medida que una comunidad es más autónoma en relación a las decisiones en torno a los recursos (de todo tipo) es menos vulnerable desde la dimensión política. Por consiguiente, a medida que una comunidad es menos independiente se hace más vulnerable. Esta dimensión de la vulnerabilidad está estrechamente vinculada con la anterior.

La vulnerabilidad 'técnica', estrechamente ligada a la vulnerabilidad física. Para WilchesChaux, podría estar en evidencia por la ausencia de diseños y estructuras sismo resistentes en un área de terremotos, por ejemplo.

La vulnerabilidad 'ideológica', está relacionada con la concepción de los hechos del devenir del mundo que tienen las personas en un grupo social. Si una comunidad, comprende en un desastre un castigo de Dios, será muy difícil que se prepare y mejore su situación. 
La vulnerabilidad 'cultural', se relaciona a la forma en que las personas se ven a sí mismos en forma individual y como parte de una comunidad. Juegan un rol muy importante los medios de comunicación, ya que contribuyen a formar las imágenes que tienen de sí mismos los individuos.

La vulnerabilidad 'educativa', la presencia (o ausencia) de conocimiento en general y más aún, sobre los posibles desastres a los que esa sociedad está sometida, sus causas, su historia, etc. generan diferentes tipos de respuestas en el comportamiento, ya sea a nivel individual o colectivo. Se puede comprender como la vulnerabilidad educativa aumenta a medida que disminuye el nivel de escolaridad y el conocimiento sobre los riesgos a los que se está expuesto. (La vulnerabilidad educativa está íntimamente relacionada a la social y a la económica).

La vulnerabilidad 'ecológica', se manifiesta cuando el modelo de desarrollo corresponde a una inapropiada y poco sustentable explotación de los recursos naturales, se tiende a incrementar la vulnerabilidad del ambiente. Como ejemplo, un territorio muy degradado ambientalmente, con una fuerte explotación de recursos naturales potencia la vulnerabilidad ambiental de las comunidades que lo habitan.

Por último, la vulnerabilidad 'institucional', refleja el nivel de posibilidades que tiene una comunidad para hacer frente a la gestión de riesgo. Se relaciona con la falta de flexibilidad de las instituciones, el exceso de burocracia, etc.

Es importante aclarar que cada una de estas vulnerabilidades constituye apenas un ángulo particular para analizar el fenómeno global. No se podría entender, por ejemplo, la vulnerabilidad física, sin considerarla una función de la vulnerabilidad económica y de la política; o ésta última sin tomar en cuenta la vulnerabilidad social, la cultural y nuevamente la económica.

Con base en esta propuesta de Wilches Chaux (1993), y entendiendo que la clasificación de las vulnerabilidades no es exhaustiva ya que pueden superponerse categorías pero que sin embargo representa un interesante instrumento para el análisis de este fenómeno de gran complejidad, en este trabajo se construyen las siguientes dimensiones combinadas, de acuerdo a los objetivos propuestos y las limitaciones del estudio (recursos, acceso a datos, entre otros): política-institucional, la socio-económica y la ambiental.

\section{MATERIALES Y MÉTODOS}

A continuación se describirán los principales aspectos metodológicos para el estudio de las dimensiones de vulnerabilidad de la cuenca.

Para analizar la vulnerabilidad ante fenómenos de origen hídrico en una cuenca en particular se recurrió a la metodología conocida como Análisis Evaluativo Multicriterio (AEM). La metodología de AEM se utiliza en situaciones donde los problemas a enfrentar son complejos en relación a la toma de decisión, tanto de la mirada privada como social . Originada en la década del ' 60 del siglo pasado, a partir de los aportes de la Teoría de la Decisión, el AEM tiene por objetivo elaborar un sistema de jerarquización de las alternativas y o proyectos. La principal ventaja que presenta esta propuesta es la incorporación de múltiples parámetros sin la necesidad de que estén expresados en el mismo atributo, en contraposición con las metodologías que requerían expresar todo en términos monetarios (Arrillaga, Grand y Bosso, 2009). Dentro de la metodología multicriterio se trabajó con el denominado Cuadro de Puntuación. Este método permite incorporar distintas dimensiones como: económica, social, ambiental, institucional, etc. atendiendo a la complejidad del problema, y luego, dentro de cada dimensión, profundizarla en variables. En este caso, se trabajó con tres (3) dimensiones y diecinueve (19) variables que se describirán más adelante. Una de las principales ventajas de este método, es que es factible combinar variables medidas en diferentes escalas como: cantidad de recursos financieros (con una escala cardinal: medida en téminos monetarios) con grado de aceptabilidad social (con una escala ordinal: alto, medio o bajo) y la disponibilidad de normativa (con una escala dicotómica). En segundo se puede introducir pesos relativos tanto a las variables como a las dimensiones, con el objetivo de obtener un indicador por sumatoria o productoria. Esta metodología permite generar un ranking o posicionamiento relativo, en este caso aplicado a determinar órdenes de vulnerabilidad territorial (dentro de la cuenca) ante fenómenos de origen hídrico.

Otros autores han aplicado a decisiones de ordenamiento territorial la mirada/perspectiva de multiples criterios, por ejemplo en el desarrollo de modelos óptimos territoriales, permitiendo 
conocer la capacidad de acogida del territorio ante una determinada actividad (Gutiérrez, Gómez y Bosque, 2010; Mastandrea y Angeles, 2020; Da Silva y Cardozo, 2015).

Contreras (2004) plantea los pasos generales a seguir para aplicar la metodología: a) definir y estructurar el problema, b) identificar las respuestas óptimas para el tomador de decisión y c) reconocer una forma que permita agregar los criterios. Estos pasos se consideraron a nivel orientativo para el desarrollo de este trabajo.

Considerando que se planteó como objetivo reconocer los órdenes de vulnerabilidad ante fenómenos de origen hídrico de la cuenca del arroyo Las Turbias, se exponen las etapas desarrolladas:

1. Identificación y construcción de las dimensiones. Se procedió a una construcción de dimensiones combinadas a partir de la tipología propuesta por Wilches Chaux (1993). Las dimensiones resultantes son tres: política-institucional, socio-económica y ambiental.

2. Selección de las variables a analizar dentro de cada dimensión. Se reconocieron en total diecinueve variables. Para cada una se elaboró una ficha, con algunos criterios esclarecedores que argumentan cómo influyen las variables en la vulnerabilidad. En la ficha se especificó: la dimensión a la que pertenece de acuerdo a lo definido en este estudio, la dimensión con la que se relaciona según Wilches-Chaux (1993), la escala de categorización de las variables (cardinal, ordinal o nominal), la justificación de la elección de la variable para comprender cómo la variación de los criterios de categorización contribuirían a la variación de la vulnerabilidad, el objetivo (se analiza cómo incide la variable en relación a la vulnerabilidad), la respuesta óptima (buscando la mínima vulnerabilidad), la fuente de datos (se indica de dónde se obtienen los datos) y la escala territorial (se indaga en las diferentes escalas territoriales que se pueden hallar (ver Tablas 1 y 2 ).

3. Recopilación de información primaria y secundaria.

4. Procesamiento de la información.

5. Elaboración de la matriz multicriterio general. Esta matriz tiene en las columnas una breve síntesis de las fichas, indicando: la escala de la variable, la dimensión (unidades), la respuesta óptima y diferentes zonas (unidades territoriales) analizadas (cuencas superior, media e inferior). Mientras que en las filas se presentan las variables (19) y las respuestas de cada una de ellas. Como primer escenario de análisis, se consideró asignar el mismo peso a cada una de las tres dimensiones (ver Tabla 3).

6. Transformación de la matriz general en una matriz estandarizada. En este paso es necesario homogeneizar las respuestas de la matriz, de tal forma que todas las respuestas sean expresadas de manera adimensional, indicando 1 para la mejor respuesta (menor vulnerabilidad) y 0 para la peor (ver Tabla 4).

7. Determinación de los valores de respuesta correspondiente a cada zona bajo análisis. Este resultado surge de ponderar el valor estandarizado de cada variable por sus pesos asignados en relación a la valoración de las dimensiones En este caso para cada zona territorial en que se subdividió la cuenca (superior, media e inferior) se obtiene un valor de vulnerabilidad para cada dimensión (ver Tabla 5).

\section{Identificación y construcción de dimensiones y variables}

En este estudio el equipo de investigación consideró como base teórica la tipología propuesta por Wiches-Chaux (1993) y se discutieron los criterios para construir las dimensiones que se abordarían para el área de estudio en particular. De acuerdo a este análisis se identificaron cuáles vulnerabilidades del autor mencionado podrían incluirse en cada una de las dimensiones determinadas.

A continuación se explican conceptualmente las dimensiones combinadas definidas que el equipo de investigadores consideró.

Se comprende como vulnerabilidad política-institucional a la incapacidad de las instituciones y entidades intermedias para desarrollar apropiadamente acciones para desempeñar su rol ante 
situaciones de riesgo hídrico. Entre las principales características se mencionan las limitaciones en: independencia para la toma de decisiones, capacidad para autofinanciarse, cantidad y calidad de recursos humanos, interinstitucionalidad, experiencia y antecedentes de la institución, normativa vigentes, gobernanza. Por ejemplo, disponer de mayores recursos económicos para afrontar el riesgo disminuye la vulnerabilidad de las instituciones. Se agrupan las dimensiones política e institucional de Wilches-Chaux (1993).

Por vulnerabilidad socio-económica, se engloba a un conjunto de factores que condicionan la vulnerabilidad ante fenómenos de origen hídrico: salud, economía, educación, pobreza, producción. Por ejemplo, en este sentido se entiende que, a medida que el indicador de Necesidades Básicas Insatisfechas (NBI) disminuye, la vulnerabilidad disminuye. Esta vulnerabilidad engloba o relaciona las dimensiones social, económica y educativa del autor mencionado.

Por último, la vulnerabilidad ambiental, considerada en este trabajo, comprende los factores ambientales estrechamente relacionados con las características físicas del territorio, naturales y antrópicas, que condicionan la vulnerabilidad. Por ejemplo, a mayor superficie de suelos con excesos hídricos, mayor vulnerabilidad. Reúne las dimensiones natural, física y de Wilches-Chaux (1993).

Posteriormente para la selección de las variables se puso énfasis en aquellas que, a criterio del equipo de investigación, describan adecuadamente la mayoría de las características de cada dimensión. A continuación se presentan las variables consideradas en las tres dimensiones:

Dimensión política-institucional:
1.1- Normativa vigente.
1.2- Estructura institucional.
1.3- Recursos humanos.
1.4- Recursos económicos.
1.5- Relación interinstitucional.
1.6- Agenda pública.
1.7- Gobernanza.
1.8- Comité de cuenca.

Dimensión socio-económica:

2.1- Población económicamente activa

2.2- Nivel de instrucción primaria.

2.3- Tenencia de terreno.

2.4- Población ocupada.

2.5- Hogares con NBI.

2.6- Capacidad de producción de la tierra.

2.7- Residentes en establecimientos agropecuarios (EAP)

Dimensión ambiental:

3.1- Pendientes topográficas.

3.2- Suelo con exceso de agua.

3.3- Densidad vial.

3.4- Densidad de drenaje.

\section{Información recopilada}

Inicialmente se realizó una búsqueda por internet en instituciones, organismos y entidades públicas y privadas (de carácter nacional, provincial, municipal, regional o local) que podrían disponer de información relacionada con la cuenca de estudio. Se estableció contacto por e-mail, en forma telefónica o personal al fin de evaluar la disponibilidad de la información.

A nivel cartográfico se pudo obtener cartas topográficas confeccionadas por el Instituto Geográfico Nacional (IGN); mapas de tipos y capacidad de suelo confeccionados por el Instituto Nacional de Tecnología Agropecuaria (INTA), el Servicio de Catastro e Información Territorial (SCIT) y al Ministerio de Infraestructura, Servicios Públicos y Hábitat (MISPH) de la provincia facilitó archivos digitales vectoriales que representan los límites políticos, vías de comunicación férrea, 
vías de comunicación vial (nacional, provincial y comunal), hidrografía, curvas topográficas, límites de cuencas, límites de parcelas catastrales y topónimos. Con respecto a imágenes satelitales se recurrió a la Comisión Nacional de Actividades Espaciales (CONAE) y a otras instituciones que ofrecen productos digitales a fin de identificar imágenes actuales del área de estudio.

Con respecto a datos estadísticos de la población, se accedió a la base de datos del Instituto Nacional de Estadística y Censos (INDEC) para acceder a los archivos vectoriales de los radios censales y al Instituto Provincial de Estadística y Censos (IPEC) donde se recopiló información de los censos poblacionales del año 2010.

En relación a la información de la normativa legal vigente relacionada a la temática de riesgo, se contactaron instituciones gubernamentales a nivel nacional, provincial y local a fin de identificar leyes, normas y decretos sancionados y reglamentados.

Entre las fuentes primarias se recurrió a las entrevistas, herramientas utilizadas en la metodología investigación cualitativa para recolectar datos no cuantificables que permiten profundizar en el análisis de ciertos aspectos que escapan a la información de censos, encuestas o informes cuantitativos. Pueden ser estructuradas, semiestructuradas y no estructuradas o abiertas. Las entrevistas semiestructuradas se basan en una guía de asuntos o preguntas y el entrevistador tiene la libertad de introducir preguntas adicionales para precisar conceptos u obtener mayor información (Sampieri et al., 2014).

Se realizaron entrevistas a integrantes de la Secretaría de Protección Civil y del MISPH de la provincia para conocer la situación de riesgo provincial. Y a nivel local se entrevistó a intendentes, presidentes comunales, responsables de los bomberos voluntarios, policía local, directores de Servicios para la Atención Médica de la Comunidad (SAMCo), integrantes de las Juntas de Protección Civil local, integrantes del Comité de cuenca del arroyo Las Turbias, productores agropecuarios de los diferentes sectores de la cuenca y vecinos de las principales localidades (Las Rosas, San Genaro, Centeno y Los Cardos).

Estas últimas entrevistas se deberían haber realizado también en las localidades de María Susana, El Trébol, Bouquet, Monte de Oca, Clason y Casalegno, al fin de obtener información que complete todos los distritos que intervienen en la cuenca del Arroyo de Las Turbias. Pero por cuestiones de tiempo y económicas relacionadas al proyecto de investigación, no se realizaron.

\section{Procesamiento de la información}

La elaboración de la cartografía base requirió la superposición de los archivos digitales recopilados. Posteriormente sobre la cartografía base se referenciaron las imágenes satelitales encontradas y se procedió a identificar y representar los caminos rurales y los canales de desagües oficiales y clandestinos.

Con los datos estadísticos se generaron planillas donde se ordenó y procesó información relacionada a las variables definidas en la dimensión socio-económica. En el caso específico de la capacidad de producción se estimó la cantidad de hectáreas de suelos A, B1 y B2 (aptitud productiva alta y media, respectivamente) en relación a la superficie de la cuenca superior, media e inferior respectivamente.

En la dimensión política-institucional, principalmente se utilizó la información recopilada en las entrevistas con el objeto de comprender la perspectiva institucional respecto a los problemas de riesgo hídrico en la cuenca o distrito administrados. Se indagó acerca de cuáles son los principales problemas, cómo actúa la estructura institucional, que recursos económicos y humanos poseen, con qué instituciones se vinculan y si se realizan evaluaciones sobre la actuación ante eventos hídricos extremos.

Las entrevistas relacionadas a los productores agropecuarios y vecinos de las localidades, se orientaron a recuperar la percepción de los actores involucrados en situaciones de riesgo hídrico. Es por ello que se indagó acerca de experiencias de inundación, si tenían recursos suficientes para afrontarlas, si recibieron ayuda del gobierno para reparación de daños, si se disponían de medios de comunicación con las autoridades de gobierno, si hubo coordinación entre instituciones responsables en la emergencia.

En la entrevista realizada al Comité de la cuenca se preguntó acerca de las acciones realizadas por el ente que administra la cuenca, las causas y consecuencias de las últimas 
crecidas, organización del Comité, equipamientos, recursos humanos y económicos, relación con los productores agropecuarios, proceso administrativo de las obras, obras ejecutadas y por ejecutar, vinculación con la provincia, fortalezas y debilidades del Comité.

Para la dimensión ambiental se obtuvo información de la cartografía generada e imágenes procesadas. Las pendientes topográficas predominantes en los diferentes sectores de la cuenca se estimaron con las curvas de nivel. Se identificaron los suelos con exceso de agua de la cuenca de acuerdo a la clasificación del INTA y se estimó la cantidad de hectáreas en relación a la superficie de cada sector de la cuenca. Para calcular la densidad vial se consideraron las longitudes de todas las vías de comunicación referidas a la superficie de cada sector. En el análisis de la variable densidad de drenaje se consideraron las longitudes de los cursos de aguas y canales oficiales y clandestinos, referidas a la superficie de cada sector.

Toda la información procesada de las fuentes primarias y secundarias se sistematizó en planillas para facilitar y ordenar el análisis de las dimensiones e implementar las matrices multicriterio.

\section{RESULTADOS Y DISCUSIÓN}

En la selección y construcción de las dimensiones de la vulnerabilidad de interés para este estudio se tomaron como base las 11 postuladas por Wilches-Chaux, combinándolas. A los efectos de establecer a qué instituciones recurrir para disponer de la información política-institucional, inicialmente se analizó la organización que existe en la provincia de Santa Fe al actuar en situaciones de riesgo hídrico. La ley de defensa civil de la provincia de Santa Fe (ley 8094) designa que los gobiernos locales deben disponer de juntas de protección civil. Como consecuencia se averiguó la existencia de las mismas y que instituciones y personas la integraban. La existencia de la junta de protección civil favorece la integración de las diferentes instituciones. También se analizó la opinión de los residentes en las partes urbanas como rurales y además al Comité de cuenca (que administra las obras a realizarse en toda la extensión de la cuenca).

Para reunir información de la dimensión socio-económica, se analizaron los datos recolectados en los censos poblacionales. Específicamente se trabajó con los últimos datos censales disponibles en la provincia del año 2010. También se recurrió a información disponible en instituciones (INTA) al fin de establecer las características productivas del suelo localizado en la cuenca de estudio. En el caso de la dimensión ambiental se recurrió a instituciones que disponen de información básica de la cuenca y se procesaron e interpretaron imágenes satelitales para disponer de datos actualizados. El análisis de vulnerabilidad se inició con la sistematización en una ficha donde se detallan los descriptores para caracterizar las variables de cada dimensión. A modo de ejemplo, en las Tablas 1 y 2 se muestran las fichas de variables de las dimensiones política-institucional y socio-económica.

\begin{tabular}{ll} 
Ficha No: & 1.1 \\
\hline Dimensión: & Política-institucional \\
\hline Variable: & Normativa vigente \\
\hline $\begin{array}{l}\text { Dimensión de Wilches- } \\
\text { Chaux a que refiere: }\end{array}$ & Política \\
\hline Justificación: & $\begin{array}{l}\text { La existencia y aplicación de normativa que avale la preparación frente al } \\
\text { riesgo, contribuye a la reducción de la vulnerabilidad. }\end{array}$ \\
\hline Objetivo: & $\begin{array}{l}\text { Reconocer la existencia y aplicación de normativas relacionadas a distintos } \\
\text { momentos del ciclo de vida de los desastres. Se trata de leyes o decretos } \\
\text { nacionales o provinciales, de ordenanzas locales, de planes de ordenamiento }\end{array}$ \\
\hline territorial, etc. \\
\hline Pscala: & Ordinal \\
\hline Resibles respuestas: & Alta, media, baja \\
\hline Fuente de la información: & Primarias: entrevistas \\
\hline Existencia de escalas & Nacional, provincial, local \\
\hline territoriales:
\end{tabular}

Tabla l. Variable normativa vigente de la dimensión política-institucional Fuente: Autores, 2021. 


\begin{tabular}{ll} 
Ficha No: & $\mathbf{2 . 1}$ \\
\hline Dimensión: & Socio-económica \\
\hline Variable: & Población económicamente activa \\
\hline $\begin{array}{l}\text { Dimensión de Wilches- } \\
\text { Chaux a que refiere: }\end{array}$ & Social \\
\hline Justificación: & $\begin{array}{l}\text { Una mayor proporción de la población económicamente activa (entre 14 y 65 } \\
\text { años) contribuye a una menor vulnerabilidad. }\end{array}$ \\
\hline Objetivo: & Reconocer la proporción de la población económicamente activa. \\
\hline Escala: & Cardinal \\
\hline Posibles respuestas: & \% \\
\hline Respuesta óptima: & Máximo \\
\hline Fuente de la información: & Censo Nacional de Población, Hogares y Viviendas 2010. \\
\hline Existencia de escalas & Provincial, local \\
territoriales: & \\
\hline
\end{tabular}

Tabla 2. Variable población económicamente activa de la dimensión socio-económica Fuente: Autores, 2021

A continuación se explican las características y los criterios de categorización utilizados en las diferentes variables de las dimensiones analizadas. En la dimensión política-institucional:

1.1- Normativa vigente: cantidad y grado de aplicación de leyes, decretos, ordenanzas reglamentadas y planes de ordenamiento territorial. A mayor cantidad de normas se considera que reduce la vulnerabilidad.

1.2- Estructura institucional: se priorizó específicamente la existencia de juntas de protección civil local. Se asume que la existencia de juntas, tiende a disminuir la vulnerabilidad.

1.3- Recursos humanos: cantidad de personal, nivel de capacitación y equipamiento disponible en instituciones relevantes (bomberos, SAMCo y destacamentos policiales). A mayor cantidad de recursos humanos, menor vulnerabilidad.

1.4- Recursos económicos: cantidad de fuentes económicas en instituciones relevantes (bomberos, SAMCo y destacamentos policiales). Mejores fuentes financieras, disminuye la vulnerabilidad.

1.5- Relación interinstitucional: periodicidad de reuniones internas, características de la comunicación con las instituciones intervinientes, disponibilidad de protocolos internos de actuación y relación con las instituciones provinciales (referido a la junta de protección civil local). Un mejor funcionamiento de la junta, disminuye la vulnerabilidad.

1.6-Agenda pública: percepción de los vecinos, en relación a la concientización implementada por las autoridades competentes, qué medios de comunicación fueron utilizados y la autoevaluación de cómo actuar ante situaciones de riesgo. Una sociedad concientizada, disminuye la vulnerabilidad.

1.7- Gobernanza: percepción de los vecinos, si se han realizado obras, si han realizado reclamos a las autoridades competentes, si han tenido acceso a ayuda económica ante situaciones de riesgo y si existen mecanismos de comunicación para la expresión de los ciudadanos. Mayor presencia del estado en situaciones de emergencia, disminuye la vulnerabilidad.

1.8- Comité de cuenca: cantidad de reuniones internas, percepción de los productores en relación a la ejecución de obras y comunicación del Comité hacia los productores, porcentaje de proyectos presentados/ejecutados. El mejor funcionamiento del Comité de cuenca disminuye la vulnerabilidad.

En las variables cuantitativas de la dimensión socio-económica:

2.1- Población económicamente activa: Se considera que la franja etaria de la población entre 14 a 65 años es potencialmente activa. A mayor población potencialmente productiva, menor vulnerabilidad; ya que podrán enfrentar de mejor forma la situación de vulnerabilidad. 
2.2- Nivel de instrucción primaria: se incluyó la población analfabeta y la que tiene únicamente educación primaria completa. Una sociedad educada, disminuye la vulnerabilidad que permitiría tomar mejores determinaciones ante la amenaza.

2.3- Tenencia de terreno: ser propietarios de inmuebles supone una mejor condición económica para enfrentar la amenaza y como consecuencia tener una menor vulnerabilidad.

2.4- Población ocupada: es la población que posee ingresos (trabaja) y sería menos vulnerable; ya que tendrá un respaldo económico ante la situación de amenaza.

2.5- Hogares con NBI: hogares que presentan necesidades básicas insatisfechas. A menor índice de NBI, menor vulnerabilidad; ya que al existir grupos de pobreza estructural tendrán mayores dificultades para mejorar la situación de vulnerabilidad.

2.6- Capacidad de producción de la tierra: se considera la superficie que tiene buena capacidad de producción del suelo, lo que proporciona una posible mejor situación económica para enfrentar la situación de amenaza. A mayor superficie productiva, menor vulnerabilidad.

2.7- Residentes en EAP: cantidad de personas que residen en los sectores de la cuenca por $\mathrm{km}^{2}, \mathrm{y}$ que pueden estar expuestos a potenciales inundaciones. A menor población residente en zonas rurales, menor vulnerabilidad.

Y en el caso de las variables de la dimensión ambiental:

3.1- Pendientes topográficas: se consideró la pendiente topográfica en cada sector de la cuenca como condición física favorable para evacuación de excedentes hídricos. A mayor pendiente del terreno, menor vulnerabilidad.

3.2- Suelo con exceso de agua: superficie de suelo que posee exceso y permanencia de agua, está expuesto a situaciones de anegamiento. A menor superficie anegada, menor vulnerabilidad.

3.3- Densidad vial: longitud de rutas principales, secundarias y caminos rurales por superficie en cada sector de la cuenca. A mayor densidad de vías de comunicación menor vulnerabilidad; ya que ante situaciones de emergencia, se poseen mejores condiciones para evacuar la población y para el ingreso de personal relacionado a instituciones de respuesta.

3.4- Densidad de drenaje: longitud de cursos de agua y canales por superficie en cada sector de la cuenca como índice facilitador del escurrimiento. A mayor densidad de drenaje, menor vulnerabilidad.

En la Tabla 3 se muestra un resumen de las variables de las tres dimensiones, especificando la escala, las unidades de medida en el caso de las escalas cardinales e indicando la respuesta óptima de la variable para disminuir la vulnerabilidad, es decir la mejor respuesta de la variable que proporciona la mejor condición de vulnerabilidad en cada sector de la cuenca.

Es pertinente aclarar que la categorización realizada en este estudio se relativiza al rango de valores de cada variable, esto significa que los resultados obtenidos posicionan la condición de vulnerabilidad en menor, media y mayor para los distintos sectores de la cuenca.

En la Tabla 4 se presenta la matriz de respuestas a la categorización inicial de las variables de acuerdo a los criterios definidos en las fichas (columnas 3,4 y 5 ) y la correspondiente matriz normalizada (columnas 6,7 y 8 ) en la cual se muestran las posiciones relativas de las variables. En color verde se destaca la condición de menor vulnerabilidad.

Si se analizan los resultados de la matriz normalizada de la Tabla 4 en relación a las variables menos vulnerables se establece que:

En la dimensión política-institucional la variable menos vulnerable es la estructura institucional (1.2). Como se mencionó anteriormente en esta dimensión no hubo datos suficientes para establecer la diferencia espacial entre los tres sectores de la cuenca.

Respecto de la dimensión socio-económica se pueden identificar distintos comportamientos en los sectores. En este sentido las variables población económicamente activa (2.1), población ocupada (2.4) y hogares con NBI (2.5) son menos vulnerables en la cuenca media, mientras que en la cuenca superior la tenencia de terreno (2.3) y la capacidad de producción de la tierra (2.6) 
presentan menor vulnerabilidad. En la cuenca inferior el nivel de instrucción (2.2) es mayor y hay menos residentes en $\operatorname{EAP}(2.7)$, lo que indica que estas variables poseen menor vulnerablidad.

\begin{tabular}{|c|c|c|c|c|c|}
\hline Dimensión & $\mathbf{N}^{\circ}$ & Variables & Escala & Unidades & Respuesta Óptima \\
\hline \multirow{8}{*}{ 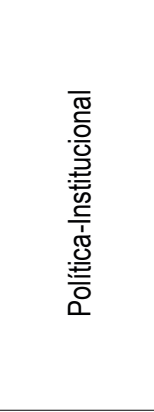 } & 1.1 & Normativa vigente & Ordinal & & alto \\
\hline & 1.2 & Estructura institucional & Ordinal & & alto \\
\hline & 1.3 & Recursos humanos & Ordinal & & alto \\
\hline & 1.4 & Recursos económicos & Ordinal & & alto \\
\hline & 1.5 & Relación interinstitucional & Ordinal & & alto \\
\hline & 1.6 & Agenda pública & Ordinal & & alto \\
\hline & 1.7 & Gobernanza & Ordinal & & alto \\
\hline & 1.8 & Comité de cuenca & Ordinal & & alto \\
\hline \multirow{7}{*}{ 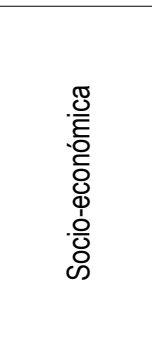 } & 2.1 & Población económicamente activa & Cardinal & $\%$ & máximo \\
\hline & 2.2 & Nivel de instrucción primaria & Cardinal & $\%$ & máximo \\
\hline & 2.3 & Tenencia de terreno & Cardinal & $\%$ & máximo \\
\hline & 2.4 & Población ocupada & Cardinal & $\%$ & máximo \\
\hline & 2.5 & Hogares con NBI & Cardinal & $\%$ & mínimo \\
\hline & 2.6 & Capacidad de producción de la tierra & Cardinal & $\%$ & máximo \\
\hline & 2.7 & Residentes en EAP & Cardinal & cant $/ \mathrm{km}^{2}$ & mínimo \\
\hline \multirow{4}{*}{ 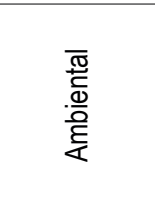 } & 3.1 & Pendientes topográficas & Cardinal & $\%$ & máximo \\
\hline & 3.2 & Suelo con exceso de agua & Cardinal & $\%$ & mínimo \\
\hline & 3.3 & Densidad vial & Cardinal & $\mathrm{km} / \mathrm{km}^{2}$ & máximo \\
\hline & 3.4 & Densidad de drenaje & Cardinal & $\mathrm{km} / \mathrm{km}^{2}$ & máximo \\
\hline
\end{tabular}

Tabla 3. Matriz original resumen de caracterización de las variables Fuente: Autores, 2021

\begin{tabular}{|c|c|c|c|c|c|c|c|}
\hline \multirow[b]{2}{*}{ Dimensión } & \multirow{2}{*}{$\begin{array}{l}\text { Var. } \\
\mathbf{N}^{\circ}\end{array}$} & \multicolumn{3}{|c|}{ Matriz de Respuestas } & \multicolumn{3}{|c|}{ Matriz Normalizada } \\
\hline & & $\begin{array}{l}\text { Cuenca } \\
\text { Superior }\end{array}$ & $\begin{array}{l}\text { Cuenca } \\
\text { Media }\end{array}$ & $\begin{array}{l}\text { Cuenca } \\
\text { Inferior }\end{array}$ & $\begin{array}{l}\text { Cuenca } \\
\text { Superior }\end{array}$ & $\begin{array}{l}\text { Cuenca } \\
\text { Media }\end{array}$ & $\begin{array}{l}\text { Cuenca } \\
\text { Inferior }\end{array}$ \\
\hline \multirow{8}{*}{ 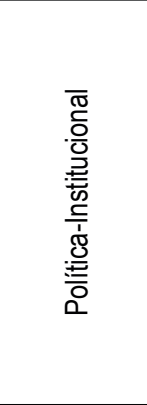 } & 1.1 & Bajo & Bajo & Bajo & 0,00 & 0,00 & 0,00 \\
\hline & 1.2 & Alto & Alto & Alto & 1,00 & 1,00 & 1,00 \\
\hline & 1.3 & Medio & Medio & Medio & 0,50 & 0,50 & 0,50 \\
\hline & 1.4 & Medio & Medio & Medio & 0,50 & 0,50 & 0,50 \\
\hline & 1.5 & Medio & Medio & Medio & 0,50 & 0,50 & 0,50 \\
\hline & 1.6 & Bajo & Bajo & Bajo & 0,00 & 0,00 & 0,00 \\
\hline & 1.7 & Medio & Medio & Medio & 0,50 & 0,50 & 0,50 \\
\hline & 1.8 & Medio & Medio & Medio & 0,50 & 0,50 & 0,50 \\
\hline \multirow{7}{*}{ 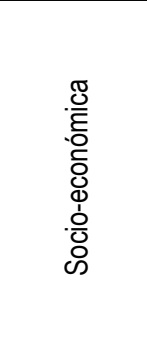 } & 2.1 & 64,70 & 70,19 & 64,13 & 0,09 & 1,00 & 0,00 \\
\hline & 2.2 & 64,67 & 62,37 & 70,21 & 0,29 & 0,00 & 1,00 \\
\hline & 2.3 & 40,02 & 37,47 & 33,78 & 1,00 & 0,59 & 0,00 \\
\hline & 2.4 & 65,06 & 71,24 & 66,48 & 0,00 & 1,00 & 0,23 \\
\hline & 2.5 & 4,75 & 3,12 & 10,12 & 0,77 & 1,00 & 0,00 \\
\hline & 2.6 & 99,48 & 98,78 & 88,15 & 1,00 & 0,94 & 0,00 \\
\hline & 2.7 & 0,66 & 0,67 & 0,52 & 0,07 & 0,00 & 1,00 \\
\hline \multirow{4}{*}{ 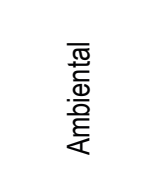 } & 3.1 & 0,11 & 0,5 & 0,15 & 0,00 & 1,00 & 0,10 \\
\hline & 3.2 & 15,61 & 25,79 & 80,28 & 1,00 & 0,84 & 0,00 \\
\hline & 3.3 & 2,64 & 2,15 & 2,55 & 1,00 & 0,00 & 0,82 \\
\hline & 3.4 & 0,331 & 0,137 & 0,487 & 0,56 & 0,00 & 1,00 \\
\hline
\end{tabular}


En cuanto a la dimensión ambiental, la cuenca media presenta la pendiente topográfica (3.1) menos vulnerable, mientras que la cuenca inferior presenta una mejor densidad de drenaje (3.4) lo que plantea menor vulnerabilidad. Además la cuenca superior posee mayor densidad vial (3.3) y menor porcentaje de suelos con exceso de agua (3.2), lo que indica menor vulnerabilidad.

Si se analizan las variables más vulnerables se destaca:

En la dimensión política-institucional las variables normativa vigente (1.1) y agenda pública (1.6). En esta dimensión no hay diferenciación entre los sectores.

En la dimensión socio-económica se presentan diferencias entre los sectores de la cuenca: en la cuenca superior es la población ocupada (2.4); en la cuenca media el nivel de instrucción primaria (2.2) y residentes en $\operatorname{EAP}(2.7)$ y en la cuenca inferior la población económicamente activa (2.1), tenencia de terreno (2.3), hogares con NBI (2.5) y capacidad de producción de la tierra (2.6).

En la dimensión ambiental la cuenca superior posee la pendiente topográfica (3.1) más baja, en la cuenca media la densidad vial (3.3) y la densidad de drenaje (3.2), mientras que en la cuenca inferior es la variable suelos con excesos de agua (3.2).

Si bien se indicaron las variables más y menos vulnerables relativas al orden de magnitud de los rangos, es necesario destacar que hay algunas variables que presentan posiciones relativas próximas entre los sectores de la cuenca, como por ejemplo:

Las cuencas superior y media se posicionan en condiciones más vulnerable respecto de la inferior, con bajos niveles de instrucción primaria (2.2) y más cantidad residentes en EAP (2.7). Poseen condiciones de menor vulnerabilidad que la cuenca inferior en las variables hogares con $\mathrm{NBI}$ (2.5), capacidad de producción de la tierra (2.6) y suelo con exceso de agua (3.2).

Los sectores superior e inferior presentan más vulnerabilidad que la cuenca media en las variables población económicamente activa (2.1), población ocupada (2.4) y pendientes topográficas (3.1). Son más favorables en cuanto a densidad vial (3.3) que la cuenca media.

Para elaborar la matriz multicriterio (ver Tabla 5) se asignó el mismo peso a las tres dimensiones, es decir $33 \%$ a cada una. Este valor se dividió por la cantidad de variables consideradas en la dimensión y se obtuvo el peso de las variables. Estos pesos multiplicados por la matriz normalizada (0-1) generó la matriz multicriterio que categoriza la vulnerabilidad.

En la Tabla 5 se observa la valoración de las variables de las tres dimensiones en cada sector de la cuenca. Las condiciones de vulnerabilidad de cada dimensión se obtuvieron mediante sumatoria de las columnas de las variables correspondientes a cada sector de la cuenca.

Se aprecia que la valoración de la vulnerabilidad política-institucional es igual en toda la cuenca como se mencionó en secciones anteriores.

Siguiendo la lógica de valoración, se deduce que la cuenca media presentaría menor vulnerabilidad socio-económica que los otros dos sectores de la cuenca. En este sentido, la vulnerabilidad ambiental es menor en la cuenca superior.

La vulnerabilidad total es la suma de los valores de las tres dimensiones. Por lo tanto, se puede inferir la diferenciación espacial relativa de la vulnerabilidad: la cuenca media es la que presentaría una menor vulnerabilidad (orden 1), la cuenca superior una vulnerabilidad media (orden 2) y la cuenca inferior con una mayor vulnerabilidad (orden 3).

A fin de visualizar espacialmente la situación de vulnerabilidad total en la cuenca de estudio se realizó un croquis con la identificación de los órdenes de vulnerabilidad mediante colores (ver Figura 2). 


\begin{tabular}{|c|c|c|c|c|c|}
\hline $\begin{array}{l}\text { Peso } \\
\text { Dimensión }\end{array}$ & $\begin{array}{l}\text { Peso } \\
\text { Variable }\end{array}$ & Variable & $\begin{array}{l}\text { Cuenca } \\
\text { Superior }\end{array}$ & $\begin{array}{l}\text { Cuenca } \\
\text { Media }\end{array}$ & $\begin{array}{l}\text { Cuenca } \\
\text { Inferior }\end{array}$ \\
\hline \multirow{8}{*}{ 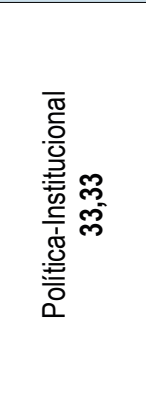 } & 4,17 & 1.1 Normativa vigente & 0,00 & 0,00 & 0,00 \\
\hline & 4,17 & 1.2 Estructura institucional & 4,17 & 4,17 & 4,17 \\
\hline & 4,17 & 1.3 Recursos humanos & 2,08 & 2,08 & 2,08 \\
\hline & 4,17 & 1,4 Recursos económicos & 2,08 & 2,08 & 2,08 \\
\hline & 4,17 & 1.5 Relación interinstitucional & 2,08 & 2,08 & 2,08 \\
\hline & 4,17 & 1.6 Agenda pública & 0,00 & 0,00 & 0,00 \\
\hline & 4,17 & 1.7 Gobernanza & 2,08 & 2,08 & 2,08 \\
\hline & 4,17 & 1.8 Comité de cuenca & 2,08 & 2,08 & 2,08 \\
\hline \multicolumn{3}{|c|}{ Vulnerabilidad Política-institucional } & 14,58 & 14,58 & 14,58 \\
\hline \multirow{7}{*}{ 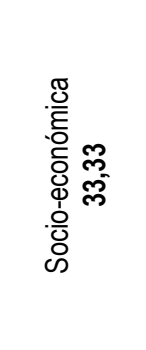 } & 4,76 & 2.1 Población económicamente activa & 0,45 & 4,76 & 0,00 \\
\hline & 4,76 & 2.2 Nivel de instrucción primaria & 1,40 & 0,00 & 4,76 \\
\hline & 4,76 & 2.3 Tenencia de terreno & 4,76 & 2,82 & 0,00 \\
\hline & 4,76 & 2.4 Población ocupada & 0,00 & 4,76 & 1,09 \\
\hline & 4,76 & 2.5 Hogares con NBI & 3,65 & 4,76 & 0,00 \\
\hline & 4,76 & 2.6 Capacidad de producción de la tierra & 4,76 & 4,47 & 0,00 \\
\hline & 4,76 & 2.7 Residentes en EAP & 0,32 & 0.00 & 4,76 \\
\hline \multicolumn{3}{|c|}{ Vulnerabilidad Socio-económica } & 15,33 & 21.57 & 10,61 \\
\hline \multirow{4}{*}{ 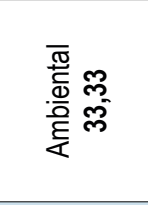 } & 8,33 & 3.1 Pendientes topográficas & 0,00 & 8,33 & 0,85 \\
\hline & 8,33 & 3.2 Suelo con exceso de agua & 8,33 & 7,02 & 0,00 \\
\hline & 8,33 & 3.3 Densidad vial & 8,33 & 0,00 & 6,80 \\
\hline & 8,33 & 3.4 Densidad de drenaje & 4,37 & 0,00 & 8,33 \\
\hline \multicolumn{3}{|c|}{ Vulnerabilidad Ambiental } & 21,33 & 15,35 & 15,99 \\
\hline \multicolumn{3}{|r|}{ Vulnerabilidad Total } & 51,24 & 51,51 & 41,18 \\
\hline \multicolumn{3}{|c|}{ Orden de menor vulnerabilidad } & 2 & 1 & 3 \\
\hline
\end{tabular}

Tabla 5. Matriz multicriterio. Categorización final

Fuente: Autores, 2021

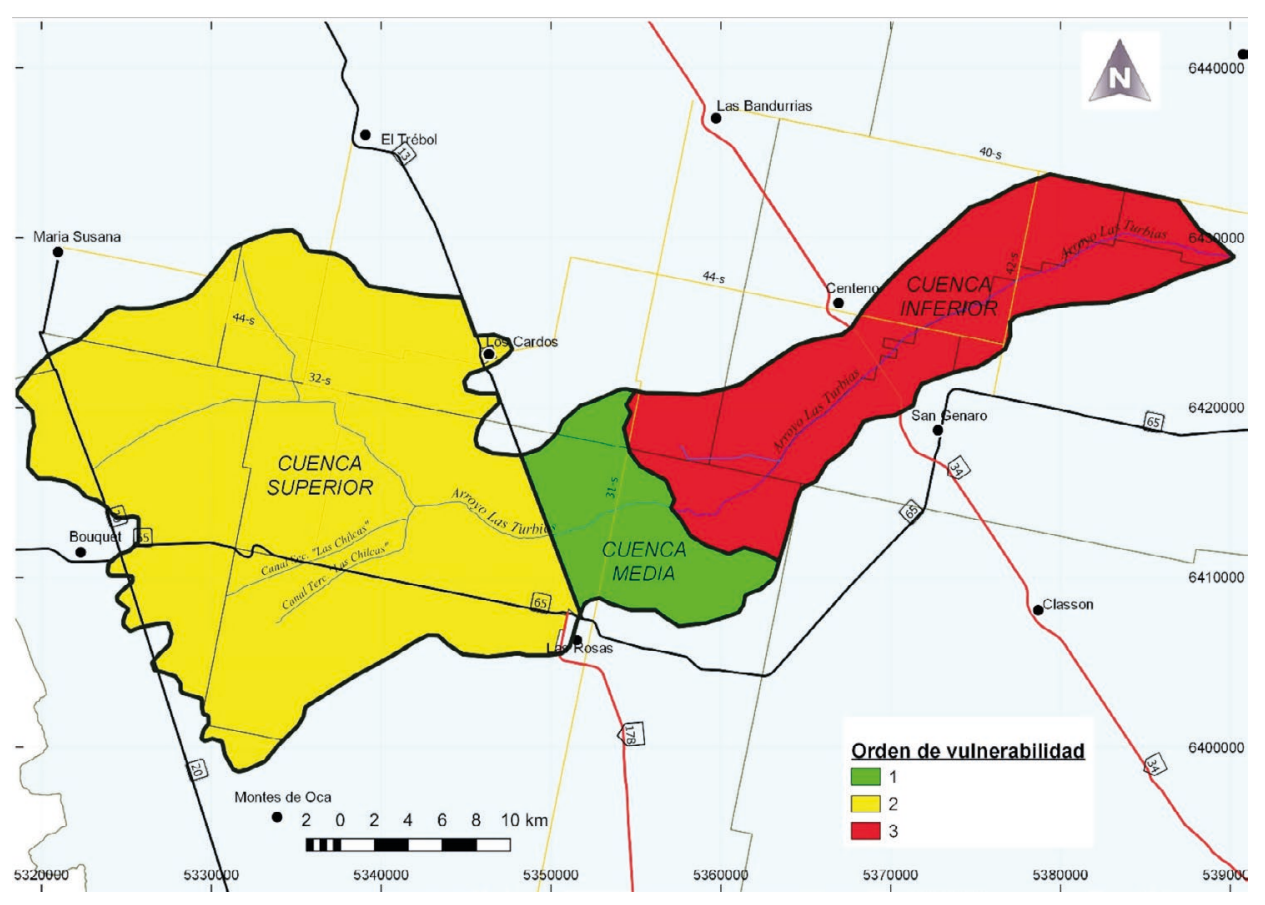

Figura 2. Vulnerabilidad total en la cuenca del arroyo Las Turbias Fuente: Autores, 2021. 


\section{CONCLUSIONES}

La metodología empleada permitió diferenciar la vulnerabilidad en los distintos sectores de la cuenca. Es factible de aplicar a un amplio rango de datos con diferentes escalas, en este caso cardinal y ordinal.

El proceso de elaboración de la matriz multicriterio requirió de la búsqueda de información de diferentes fuentes. Para algunas variables se valoró principalmente la información proveniente de las entrevistas realizadas a los referentes de las instituciones (intendentes, presidentes comunales, responsables de bomberos, SAMCo y destacamentos policiales, responsables en el Comité de cuenca) encargadas de gestionar el riesgo ante fenómenos de origen hídrico en la cuenca.

Asimismo se consideró valiosa la opinión de vecinos de localidades y productores de los diferentes sectores de la cuenca ya que permitió corroborar la información brindada por los referentes de las instituciones.

Respecto a la información secundaria se dispuso de los censos 2010 (última actualización), la cual se procesó con el fin de categorizar las variables socio-económicas.

La matriz multicriterio se obtuvo a partir del análisis de 19 variables de tres dimensiones seleccionadas para este estudio.

En cada sector de la cuenca se estableció un orden relativo de vulnerabilidad de cada dimensión y de la vulnerabilidad total.

Los resultados indican diferencias de vulnerabilidad entre los distintos sectores de la cuenca para algunas dimensiones. En la dimensión política-institucional el análisis se realizó con información de los cuatro distritos principales de la cuenca (Las Rosas, Los Cardos, San Genaro y Centeno), ya que no se dispuso de información en los demás distritos que integran la cuenca. Por ello los resultados de esta dimensión son iguales para los tres sectores de la cuenca.

Las dimensiones socio-económica y ambiental presentan diferencias entre los sectores de la cuenca, ya que se procesaron datos censales distritales y regionales para cada superficie del sector.

En referencia a los valores de vulnerabilidad total, al considerar la valoración de las tres dimensiones, no se observa grandes variaciones en cada sector de la cuenca. Se deduce inicialmente que la cuenca inferior presenta condiciones que podrían aumentar la vulnerabilidad.

Este análisis proporciona información valiosa para los tomadores de decisión porque permite identificar las variables sobre las cuáles gestionar para disminuir la vulnerabilidad.

En este trabajo se consideró un primer escenario asignando el mismo peso a cada dimensión, Como investigaciones futuras es deseable que se analicen otros escenarios que atribuyan diferentes pesos a cada dimensión y a cada variable dentro de cada dimensión, lo que conducirá a identificar las variables más sensibles a la vulnerabilidad.

Asimismo se espera que del análisis realizado se puedan generar inquietudes para definir otras variables representativas del territorio que no se han considerado en esta investigación y que como proceso dinámico contribuyan a la toma de decisiones.

\section{AGRADECIMIENTOS}

Los autores de este trabajo agradecen a los estudiantes de la Facultad de Ingeniería y Ciencias Hídricas (FICH-UNL) Andrea Cerri, Gabriel Cabrol y Sofía Francovich por los aportes realizados en el procesamiento de datos.

\section{REFERENCIAS}

Arrillaga, H., Grand, M.L., \& Busso, G. (2009). Vulnerabilidad, riesgo y desastres. Sus relaciones de causalidad con la exclusión social en el territorio urbano santafesino. En: Herzer, H., \& Arrillaga, H. (Eds.), La construcción social del riesgo y el desastre en el aglomerado Santa Fe (pp.59-104). Universidad Nacional del Litoral.

Blaikie, P., Cannon, T., Davies, I., \& Wisner, B. (1996). Vulnerabilidad. El entorno social, político y económico de los desastres. La Red.

Castignani, H. (2011). Zonas agroeconómicas homogéneas. Instituto Nacional de Tecnología Agropecuaria. Disponible en https://inta.gob.ar/sites/default/files/ script-tmp-inta-_zah_santa_fe.pdf 
Contreras, E. (2004). Serie Manuales 37 Evaluación social de inversiones públicas: enfoques alternativos y su aplicabilidad para Latinoamérica. CEPAL.

Cutter, S. (2006). Hazard vulnerability and environmental justice. Earthscan.

Cutter, S., Barnes, L., Berry, M., Burton, C., Evans, E., Tate, E., \& Webb, J. (2008). Place-based model for understanding community resilience to natural disasters. Global Environmental Change, 18, 598-606.

Da Silva, C., \& Cardozo, O. (2015). Evaluación multicriterio y Sistemas de Información Geográfica aplicados a la definición de espacios potenciales para uso del suelo residencial en Resistencia (Argentina). GeoFocus, 16, 23-40.

Documento País. (2012). Riesgo de desastres en la Argentina. UNDP \& Cruz Roja Argentina.

Foschiatti, A. (2009). Aportes conceptuales y empíricos de la vulnerabilidad global. Editorial Universitaria de la Universidad Nacional del Nordeste.

Gardiol, M., Cardoso, M., Finelli, N., Grand, L., \& Morresi, M. (2018). Análisis preliminar de la vulnerabilidad institucional ante fenómenos de riesgo hídrico en la cuenca del arroyo Las Turbias (Provincia de Santa Fe). En XII Jornadas de investigación en geografía. Facultad de Humanidades y Ciencias, Universidad Nacional del Litoral, Santa Fe, Santa Fe, Argentina.

Gobierno de Santa Fe. (2012). Atlas de riesgo por inundaciones de la provincia de Santa Fe. Gobierno de Santa Fe.

Gutiérrez Angonese, J., Gómez Delgado, M., \& Bosque Sendra, J. (2010). Simulación de crecimiento urbano mediante evaluación multicriterio y TIG en el Gran San Miguel de Tucumán (Argentina). En Ojeda, J., Pita, M., \& Vallejo, I. (Eds.), Tecnologías de la Información Geográfica: La Información Geográfica al servicio de los ciudadanos (pp. 873-888). Secretariado de Publicaciones de la Universidad de Sevilla.

Lavell, A. (2004). Antecedentes, formación y contribución al desarrollo de conceptos, estudios y la práctica en el tema de los riesgos y desastres en América Latina: 1980-2004. La Red.

Mastrandrea, A., \& Angeles, G. (2020). Evaluación multicriterio aplicada a la determinación de escenarios. Cuadernos Geográficos, 6o(1), 181-202.

Renda, E., Rozas Garay, M., Moscardini, O., \& Torchia, N. (2017). Manual para la elaboración de mapas de riesgo. PNUD \& Ministerio de Seguridad de la Nación.

Sampieri, R., Fernández Collado, C., \& Baptista Lucio. M. (2014). Metodología de la Investigación. McGraw-Hill \& Interamericana Editores.

Vargas, J. (2002). Políticas públicas para la reducción de la vulnerabilidad frente a los desastres naturales y socio-naturales. Serie Medio Ambiente CEPAL, 5o, 159.

Wilches-Chaux, G. (1993). La vulnerabilidad global. En Maskrey, A. (Ed.), Los Desastres No Son Naturales (pp.11-44). La Red. 